\title{
A Space-Time Block-Coded OFDM Scheme for Unknown Frequency-Selective Fading Channels
}

\author{
Murat Uysal, Student Member, IEEE, Naofal Al-Dhahir, Senior Member, IEEE, and \\ Costas N. Georghiades, Fellow, IEEE
}

\begin{abstract}
We introduce a space-time block-coded orthogonal frequency-division multiplexing (STBC-OFDM) scheme for frequency-selective fading channels which does not require channel knowledge either at the transmitter or at the receiver. The decoding algorithm is based on generalized maximum-likelihood sequence estimation. We investigate the performance of the proposed scheme over two-tap Rayleigh fading channels. Simulation results show the performance to be near optimum.
\end{abstract}

Index Terms-Frequency-selective fading channels, maximumlikelihood sequence estimation, OFDM, space-time coding.

\section{INTRODUCTION}

$\mathbf{S}$ PACE-TIME block coding (STTC) was introduced in [1] and [2] as an effective transmit diversity technique to combat fading. For a fixed number of transmit antennas, its decoding complexity increases exponentially with the transmission rate. Since space-time codes are originally designed for flat-fading channels, it is challenging to apply them over frequency-selective channels. One approach is to employ orthogonal frequency-division multiplexing (OFDM) which converts a frequency-selective channel into parallel independent frequency-flat subchannels using the computationally efficient fast Fourier transform (FFT). OFDM has been applied to space-time coding in [3], however, perfect channel state information (CSI) is assumed available at the receiver. Recently, in [4], a simple and efficient receiver structure has been proposed for STBC schemes over frequency-flat fading channels, which does not require any kind of channel knowledge.

In this letter, we extend the receiver structure in [4] for frequency-selective channels by combining it with OFDM signaling. The outline of the letter is as follows: We start in Section II by describing the system model. In Section III, the decoding rule is derived, from which a recursive expression is developed for practical implementation. We present the performance of the introduced receiver in Section IV and finally conclude in Section V.

\section{SYSTEM MODEL}

The block diagram of STBC-OFDM is illustrated in Fig. 1. The binary data stream is first modulated and mapped to a

Manuscript received April 28, 2001. The associate editor coordinating the review of this letter and approving it for publication was Dr. H. Sari.

M. Uysal and C. N. Georghiades are with the Department of Electrical Engineering, Texas A\&M University, College Station, TX 77843 USA (e-mail uysal@ee.tamu.edu; georghia@ee.tamu.edu).

N. Al-Dhahir is with the AT\&T Shannon Laboratory, Florham Park, NJ 07932 USA (e-mail: naofal@research.att.com).

Publisher Item Identifier S 1089-7798(01)09047-0.

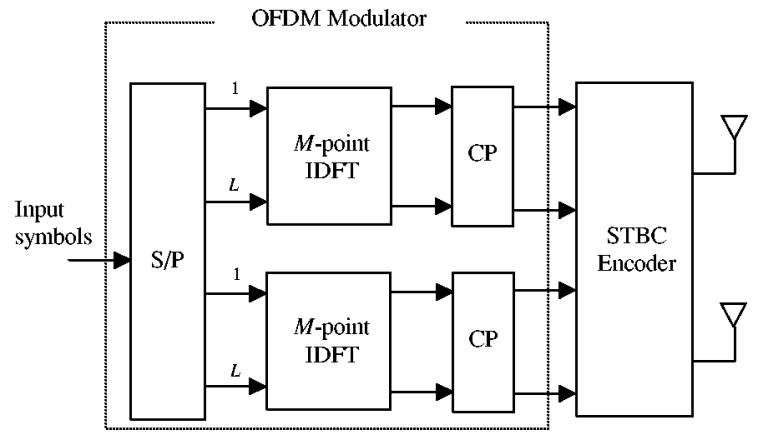

(a)

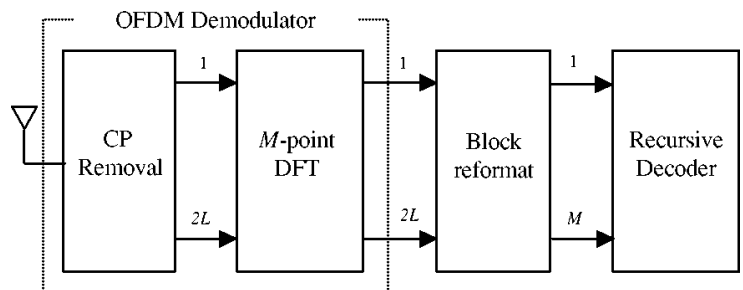

(b)

Fig. 1. (a) Transmitter structure. (b) Receiver structure.

sequence of complex modulation symbols. The modulated sequence is then passed through a serial-to-parallel converter producing $L$ data streams of length $M$ in each transmit branch. An $M$-point Inverse discrete Fourier transform (IDFT) is performed on each serial data stream. The output of the IDFT block is $M$ time-domain samples, corresponding to an OFDM frame. To avoid the effects of intersymbol-interference, a cyclic prefix (CP) of length $\nu$ is added to output samples, resulting in a frame of length $M+\nu$. Then, each OFDM frame is fed into a space-time block encoder. Although our approach is applicable to any space-time block code from the family of orthogonal designs [2], we use Alamouti's scheme [1] for simplicity. The $l$ th (out of $L$ ) OFDM frames transmitted from the first and second antennas, respectively, are given by

$$
\begin{aligned}
& \left(X_{l}^{1}(0) \ldots X_{l}^{1}(M-1)\right) \\
& \stackrel{I D F T}{\longrightarrow}\left(x_{l, 0}^{1} \ldots x_{l, M-1}^{1}\right) \\
& \stackrel{C P}{\longrightarrow}\left(x_{l, M-\nu}^{1} \ldots x_{l, M-1}^{1}, x_{l, 0}^{1} \ldots x_{l, M-1}^{1}\right) \\
& \left(X_{l}^{2}(0) \ldots X_{l}^{2}(M-1)\right) \\
& \stackrel{I D F T}{\longrightarrow}\left(x_{l, 0}^{2} \ldots x_{l, M-1}^{2}\right) \\
& \stackrel{C P}{\longrightarrow}\left(x_{l, M-\nu}^{2} \ldots x_{l, M-1}^{2}, x_{l, 0}^{2} \ldots x_{l, M-1}^{2}\right) .
\end{aligned}
$$


For the next frame interval, the OFDM frame

$$
\left(x_{l, M-\nu}^{1^{*}} \ldots x_{l, M-1}^{1^{*}}, x_{l, 0}^{1^{*}} \ldots x_{l, M-1}^{1^{*}}\right)
$$

is transmitted from the second antenna, and

$$
\left(-x_{l, M-\nu}^{2^{*}} \cdots-x_{l, M-1}^{2^{*}},-x_{l, 0}^{2^{*}} \cdots-x_{l, M-1}^{2^{*}}\right)
$$

is transmitted from the first antenna according to the signaling structure of Alamouti's scheme, where $(\cdot)^{*}$ denotes complex conjugation. In our model, we represent each of the channels from the two transmit antennas to the receive antenna by a finite-impulse-response filter with memory $\nu$. At the receiver, first the cyclic prefix is removed, then the received signal is passed through a DFT operation. It can be shown that the output of the OFDM demodulator is given by

$$
\begin{gathered}
Y_{l}(k)=\sum_{m=1}^{2} X_{l}^{m}(k) H^{m}(k)+N_{l}(k), \\
l=1 \ldots 2 L, k=0, \ldots, M-1
\end{gathered}
$$

where $N_{l}(k)$ are independent samples of a Gaussian random variable with variance $N_{0}$ and $H^{m}(k)$ is the frequency response of the channel from the $m$ th transmit antenna to the single receive antenna at the $k$ th tone. After the DFT operation, the receiver constructs a stream of $2 L$ frequency-domain samples for each tone, taking the $k$ th frequency component from each frame. Therefore, $2 L$ streams, each of length $M$, are reformatted into a total of $M$ streams each of length $2 L$ [Fig. 1(b)]. The resulting streams are fed to the detection algorithm whose structure is detailed in the next section. Taking into account the underlying coding structure, we define the following sub-matrices, $i=1,2, \ldots, L$ :

$$
\boldsymbol{Y}_{i}(k)=\left(\begin{array}{c}
Y_{2 i-1} \\
Y_{2 i}
\end{array}\right), \boldsymbol{X}_{i}(k)=\left(\begin{array}{cc}
X_{i}^{1}(k) & X_{i}^{2}(k) \\
-X_{i}^{2^{*}}(k) & X_{i}^{1^{*}}(k)
\end{array}\right) .
$$

Furthermore defining $\boldsymbol{Y}(k)=\left[\boldsymbol{Y}_{1}(k), \ldots, \boldsymbol{Y}_{L}(k)\right]^{T}, \boldsymbol{X}(k)=$ $\left[\boldsymbol{X}_{1}(k), \ldots, \boldsymbol{X}_{L}(k)\right]^{T}, \boldsymbol{H}(k)=\left[H^{1}(k), H^{2}(k)\right]^{T}, \boldsymbol{N}(k)=$ $\left[\boldsymbol{N}_{1}(k), \ldots, \boldsymbol{N}_{2 L}(k)\right]^{T_{1}}$, we can rewrite the decoder input in a compact matrix notation as

$$
\boldsymbol{Y}(k)=\boldsymbol{X}(k) \boldsymbol{H}(k)+\boldsymbol{N}(k) .
$$

\section{DECODING ALGORITHM}

The decoder is based on generalized maximum-likelihood sequence estimation [5], which is in general a two-step process. For each frequency tone $k, k=0, \ldots, M-1$, the decoding rule is based on the maximization rule

$$
\begin{aligned}
& \hat{\boldsymbol{X}}(k)=\arg \max _{X(k)} \ln p(\boldsymbol{Y}(k) \mid \boldsymbol{X}(k), \hat{\boldsymbol{H}}(k)) \text { with } \\
& \hat{\boldsymbol{H}}(k)=\arg \max _{H(k)} \ln p(\boldsymbol{Y}(k) \mid \boldsymbol{X}(k), \boldsymbol{H}(k))
\end{aligned}
$$

where the logarithmic likelihood function is given as

$$
\ln p(\boldsymbol{Y}(k) \mid \boldsymbol{X}(k), \boldsymbol{H}(k))=-\|\boldsymbol{Y}(k)-\boldsymbol{X}(k) \boldsymbol{H}(k)\|^{2}
$$

\footnotetext{
${ }^{1}$ Throughout this paper, we use $(\cdot)^{T}$ and $(\cdot)^{\prime}$ for the transpose and transpose conjugate operations, respectively. Bold face letters are used to represent matrices.
}

Maximizing (5) with respect to $\boldsymbol{H}(k)$ yields the channel estimate (i.e., the $k$ th DFT coefficient of the channel impulse response),

$$
\hat{\boldsymbol{H}}(k)=\left(\boldsymbol{X}^{\prime}(k) \boldsymbol{X}(k)\right)^{-1} \boldsymbol{X}^{\prime}(k) \boldsymbol{Y}(k) .
$$

For Alamouti's scheme, or space-time block codes based on orthogonal designs in general, the first term in (6) turns to be a scaled identity matrix; therefore (6) reduces to

$$
\hat{\boldsymbol{H}}(k)=\boldsymbol{X}^{\prime}(k) \boldsymbol{Y}(k) \text {. }
$$

Inserting the channel estimate in (5) yields the simple decoding rule

$$
\hat{\boldsymbol{X}}(k)=\arg \max _{X(k)}\left\|\boldsymbol{X}^{\prime}(k) \boldsymbol{Y}(k)\right\|^{2}, k=0,1, \ldots, M-1
$$

where $\hat{X}(k)$ consists of the estimates for the $k$ th component in each OFDM frame. Collecting the outputs of all $M$ processors, we can construct the estimates for the OFDM frames transmitted from both antennas. It is clear from the form of the decoding rule that it results in a phase ambiguity. The ambiguity can be resolved by setting the first samples of the decoder inputs to a specific value. This requires the first OFDM frame from each of the two antennas to be constructed from pilot symbols. Direct implementation of (8) is clearly impractical due to its substantial computational complexity. However, it is possible to develop a recursive expression from (8). Expanding (8) and using the sub-blocks defined in (2), we can obtain a recursive form as

$$
\begin{aligned}
& \lambda^{i}(\boldsymbol{X}(k))=\lambda^{i-1}(\boldsymbol{X}(k)) \\
& \quad+\operatorname{Re}\left\{\boldsymbol{Y}_{i}^{\prime}(k) \boldsymbol{X}_{i}(k)\left(\sum_{j=\max (1, i-J)}^{i-1} \boldsymbol{X}_{j}^{\prime}(k) \boldsymbol{Y}_{j}(k)\right)\right\} .
\end{aligned}
$$

The inner term above can be interpreted as a channel estimate based on a truncation interval of $J$ sub-blocks. It should be noted that, in practice, the pilot symbols inserted at the beginning of the frames to resolve the phase ambiguity can be also used to improve the quality of the channel estimate. For this purpose, a slight modification in the recursive expression should be made as to take the inner term as an average over the estimate obtained from previous blocks and the estimates obtained from the pilot symbols. For example, assume that truncation is restricted to just the previous sub-block (i.e., $J=1$ ), then the metric expression should be modified as

$m t= \begin{cases}\operatorname{Re}\left\{\boldsymbol{Y}_{\mathrm{i}}^{\prime}(\mathrm{k}) \boldsymbol{X}_{\mathrm{i}}(\mathrm{k})\left(\hat{\boldsymbol{H}}_{\mathrm{i}-1}(\mathrm{k})+\hat{\boldsymbol{H}}_{\mathrm{L}+1}(\mathrm{k})\right)\right\}, & \mathrm{i}=2 \\ \operatorname{Re}\left\{\boldsymbol{Y}_{i}^{\prime}(k) \boldsymbol{X}_{i}(k)\left(\hat{\boldsymbol{H}}_{i-1}(k)+\hat{\boldsymbol{H}}_{l}(k)+\hat{\boldsymbol{H}}_{L+1}(k)\right)\right\}, & \mathrm{i}>2\end{cases}$

where $\hat{\boldsymbol{H}}_{i-1}(k)=\hat{\boldsymbol{X}}_{i-1}^{\prime}(k) \hat{\boldsymbol{Y}}_{i-1}(k)$ is the estimate for the $k$ th DFT coefficient of the channel impulse response based on previous sub-block $\hat{\boldsymbol{H}}_{1}(k)$ and $\hat{\boldsymbol{H}}_{L+1}(k)$ are the channel estimates based on the pilot symbols inserted at the beginnings of the current and next frames respectively. Taking larger truncation intervals will obviously improve the performance; however this comes at the price of increasing decoding complexity (i.e., large number of trellis states). This can be especially prohibitive if higher order modulation schemes are used. Also, using a longer window may make the assumption that the channel is static over it (an assumption made in deriving the receiver) less likely to be true. Therefore, keeping the decoder complexity minimal, we employ an alternative iterative approach to further improve performance: first GMLSE technique with possible minimal com- 


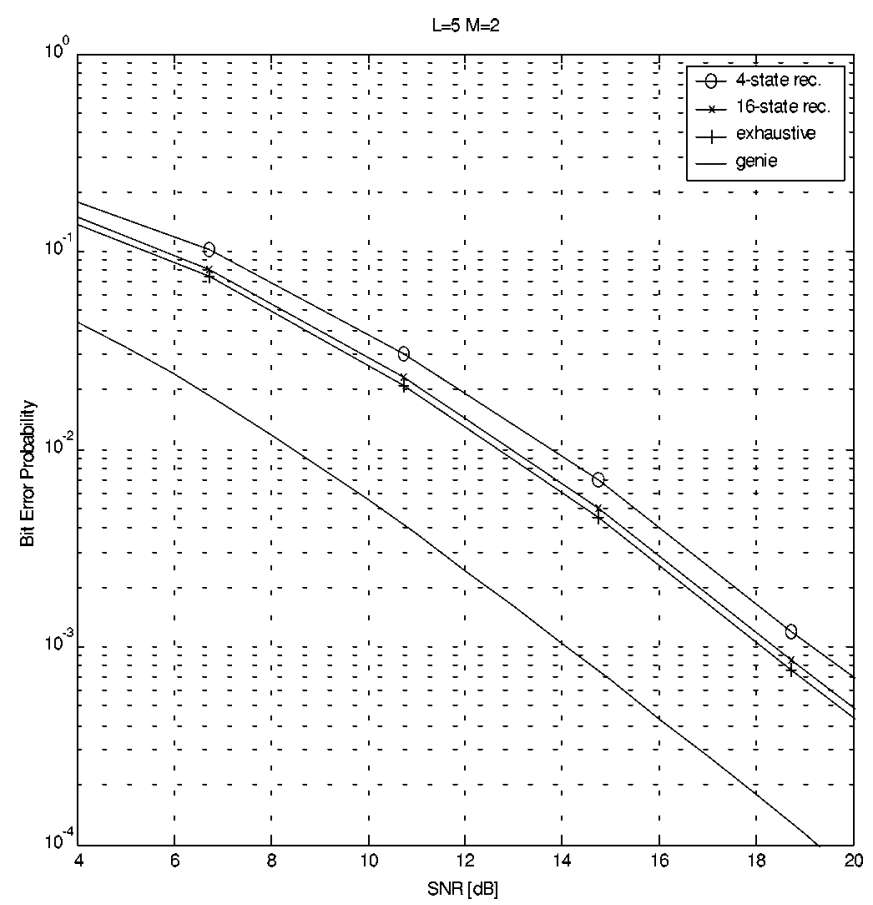

Fig. 2. Optimum versus recursive decoder performance.

plexity is used and initial data estimates are obtained; then a channel estimate is computed based on the whole decoded data and used in the coherent receiver. The resulting decoded data can be then used in refining the channel estimate.

\section{PERFORMANCE}

We investigate the performance of the proposed scheme employing BPSK modulation over a two-tap quasi-static Rayleigh fading channel. First, we compare the performance of the proposed recursive decoder to that of the optimum decoder based on an exhaustive search. For this purpose, a signaling scheme with parameters $L=5$ and $M=2$ is chosen. The exhaustive search results, based on the maximization of (8), are shown in Fig. 2. Even for this simple case, the exhaustive search requires a search among $2^{16}=65536$ possible candidates. In the figure, besides the exhaustive search, recursive expression (9) implemented with 4-state and 16-state trellises (corresponding to truncation to previous one and two sub-blocks, respectively) are shown as well. The recursive decoder achieves a near-optimum performance: it performs within $1 \mathrm{~dB}$ of the optimum decoder and this gap further decreases using a 16-state trellis. For comparison purposes, we also included the performance of Alamouti's scheme for the case of perfect CSI, which may be considered as a "genie" bound. It should be also emphasized that in the performance curves shown, SNR is penalized by the loss resulting from the pilot insertion rate and $\mathrm{CP}$ extension. Due to the unrealistic choices of $M$ and $L$ in the above scenario (made in order to make the exhaustive search feasible) the loss is high, resulting in a poor overall performance compared to the genie bound. In the following we focus on the case with practical pa-

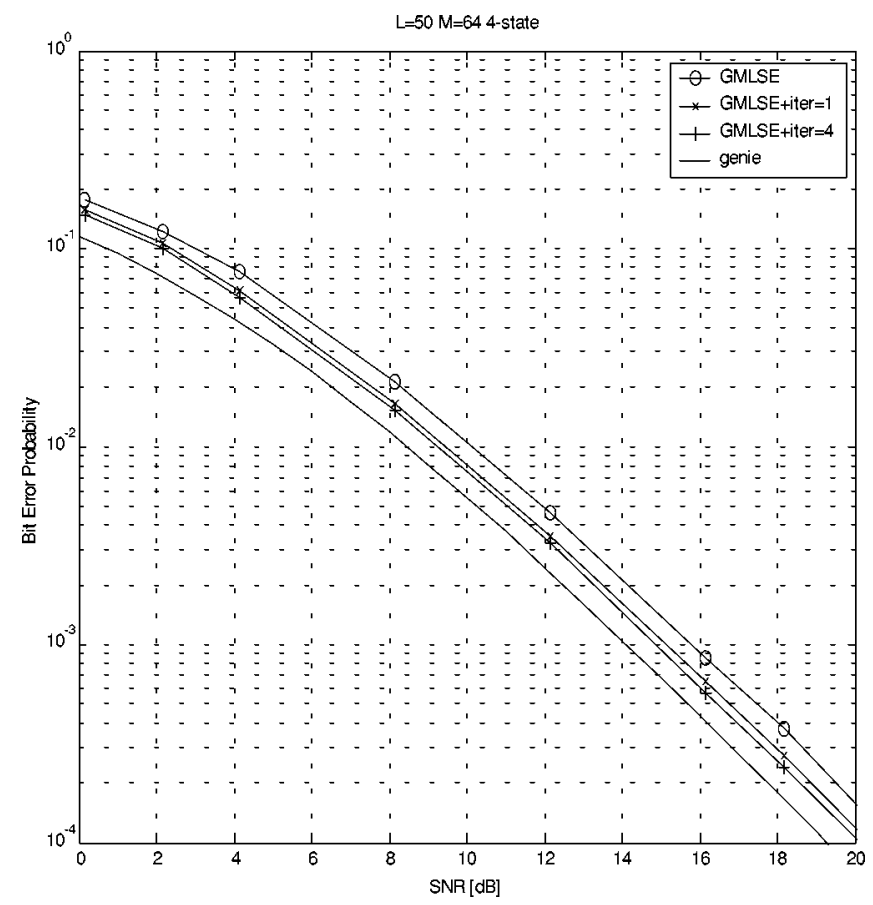

Fig. 3. Performance with modified metric and iteration.

rameter choices $L=50, M=64$ and assume a 4-state trellis in the decoder with the modified branch metric. Under these assumptions, the performance results are illustrated in Fig. 3. It is seen that the performance of the proposed receiver is within $1.5 \mathrm{~dB}$ of the genie bound. Further improvement is obtained through the iterative procedure with only a small number of iteration steps. It is also observed that most of the performance gain comes from the first iteration step.

\section{CONCLUSION}

We proposed a STBC-OFDM scheme for frequency-selective fading channels. The scheme does not require CSI either at the transmitter or at the receiver. The decoding rule is based on GMLSE, whose form allows the derivation of a recursive expression. Simulation results show the performance of the proposed recursive-type receiver to be near optimum.

\section{REFERENCES}

[1] S. M. Alamouti, "A simple transmit diversity technique for wireless communications," IEEE J. Select. Areas Commun.., vol. 16, pp. 1451-1458, Oct. 1998.

[2] V. Tarokh, H. Jafarkhani, and A. R. Calderbank, "Space-time block codes from orthogonal designs," IEEE Trans.Inform. Theory, vol. 45, pp. 1456-1467, July 1999.

[3] D. Agrawal, V. Tarokh, A. Naguib, and N. Seshadri, "Space-time coded OFDM for high data rate wireless communication over wideband channels," in Proc. IEEE VTC'98, 1998, pp. 2232-2236.

[4] M. Uysal and C. N. Georghiades, "Efficient implementation of a maximum likelihood detector for space-time coded systems," in Proc. Allerton Conf., Oct. 2000.

[5] R. Raheli, A. Polydoros, and C. Tzou, "Per-survivor processing: A general approach to MLSE in uncertain environments," IEEE Trans. Commun., vol. 43, pp. 354-364, Feb.-Apr. 1995. 